\title{
PROPUESTA PARA LA INTEGRACIÓN DE SISTEMAS DE GESTIÓN EN EL TECNOLÓGICO DE DURANGO
}

\section{PROPOSAL FOR THE INTEGRATION OF MANAGEMENT SYSTEMS IN THE TECNOLÓGICO DE DURANGO}

Recibido: $1^{\circ}$ de noviembre de 2021

Aceptado: 2 de diciembre de 2021
O.A. García Cano ${ }^{1}$

A. Ramírez Márquez ${ }^{2}$ C.O. Ríos Orozco ${ }^{3}$

\section{RESUMEN}

Basados en la metodología de Gallego (2013), se presentan los avances en la implementación de un sistema de gestión integrado, para el Instituto Tecnológico de Durango, en el cual se conjunten a los sistemas actualmente certificados: calidad y ambiental, otras normas como: educativa, salud y energía. Se revisaron los procedimientos claves de los procesos del Sistema de Calidad.

PALABRAS CLAVE: Sistema de gestión integrado, implementación, certificación, procesos.

\begin{abstract}
Based on the methodology of Gallego (2013), the advances in the implementation of an integrated management system are presented, for the Technological Institute of Durango, in which the currently certified systems are combined: quality and environmental, other standards such as: educational, health and energy. The key procedures of the Quality System processes were reviewed.
\end{abstract}

KEY WORDS: Integrated management system, implementation, certification, process.

\section{INTRODUCCIÓN}

En la actualidad existe una gran necesidad de estandarizar los procesos educativos, además de realizar la incorporación de normatividad internacional a las actividades cotidianas desarrolladas al interior de las instituciones educativas, es por ello que Hurtado (2008) indica que resulta muy conveniente el trabajar como un solo equipo para lograr la integración de los sistemas de gestión, de manera que los esfuerzos se unifiquen para lograr mejores resultados (p. 16). Es por ello que, en el Instituto Tecnológico de Durango se realiza el esfuerzo para lograr un sistema de gestión integrado, que comprenda la normatividad del sistema de gestión de calidad, ambiental, energético, educativo, de seguridad y salud en el trabajo (Gallego, 2013, p. 15). Cabe señalar que actualmente se cuenta con dos sistemas de gestión, el sistema de gestión de calidad de manera individual y el sistema de gestión ambiental en el formato de multisitios, con lo que se tiene la columna vertebral para la migración a un sistema de gestión integrado en individual, que permita al Tecnológico demostrar su compromiso a hacia la calidad, el ambiente, la seguridad, la salud, el ahorro energético y el compromiso educativo.

\section{METODOLOGÍA}

El organismo ISO, es decir, la Organización Internacional para la Estandarización (International Organization for Standarization) que se fundó en 1946 con el fin de crear un

\footnotetext{
${ }^{1}$ Profesor de tres cuartos de tiempo. Tecnológico Nacional de México, Campus Durango, obedgarciac@itdurango.edu.mx

${ }^{2}$ Profesor de tiempo completo. Tecnológico Nacional de México, Campus Durango, aramirez@itdurango.edu.mx

${ }^{3}$ Profesor de asignatura. Tecnológico Nacional de México, Campus Durango, crios@itdurango.edu.mx 
conjunto común de normas para la manufactura, el comercio y las comunicaciones. Ha permitido estandarizar a nivel mundial muchas organizaciones de diversas áreas, cada una de ellas tiene diferentes giros, pero todos buscan entrar en el círculo de la mejora continua. Según la encuesta ISO Survey de 2019, en todo el mundo existen 883,521 empresas certificadas bajo normatividad ISO 9001, y el sistema más implementado, es el sistema de gestión de calidad, enseguida, aparece la norma ISO 14001, como el sistema de gestión que se enfoca al cuidado ambiental, y en tercer lugar, aparece el ISO 45001, el cual debido a la época que se está viviendo ha cobrado muchísima relevancia.

Sin embargo, a pesar de que cada vez más empresas busquen la certificación, es importante destacar que lograrlo no es nada sencillo. Como bien reconoce Gallego (2013) implantar un sistema de gestión no es camino de rosas, sino un camino lleno de escollos (p. 16) es por ello que sugiere se realicen los siguientes seis pasos:

- Un planteamiento correcto desde el inicio.

- Convencimiento de la dirección.

- Nombrar una persona encargada únicamente de esta función.

- Redactar documentos útiles, sencillos y fácilmente entendibles.

- No generar burocracia.

- Marcarse objetivos claros a corto/medio/largo plazo.

Es por ello que en consonancia con el método expuesto por Gallego, se comienza determinando el alcance del sistema de gestión integrado. Utilizando para ello cinco normas para su adecuación y consecución. Las normas elegidas son ISO 9001:2015 de sistemas de gestión de la calidad, ISO 14001:2015 de sistemas de gestión ambiental, la ISO 21001:2018 Sistemas de gestión educativa, la ISO 45001:2018 sistemas de gestión energética y la ISO 5001:2018 Sistema de gestión de seguridad y salud en el trabajo. La norma ISO 9001:2015 aplicada a sistemas de gestión de la calidad es la norma con la que se debe partir, puesto que la calidad, es decir, el grado inherente del cumplimiento de requisitos es la norma de partida, es lo prioritario a la hora de comenzar a integrar sistemas.

Los beneficios principales que ya se tienen son como los enuncia Serrano (2018), mejora la atención del cliente, realza el enfoque a procesos, identifica las áreas de oportunidad, genera procesos estandarizados en la organización educativa y fomenta la confianza de los entes implicados (p. 15). En la actualidad ya se tienen declarados cinco procesos fundamentales en el proceso educativo: académico, vinculación, administración de recursos, planeación y calidad.

En la figura 1, se observa que actualmente la institución cuenta con algunos certificados en los sistemas de gestión, el certificado actual de forma individual es el ISO 9001:2015, en el proceso educativo. 

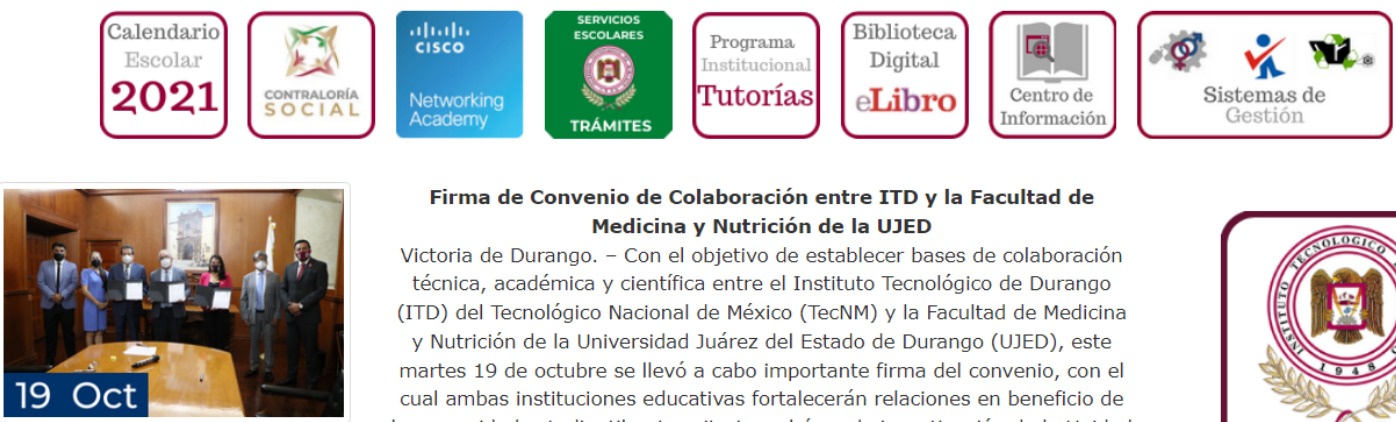

Firma de Convenio de Colaboración entre ITD y la Facultad de Medicina y Nutrición de la UJED

Victoria de Durango. - Con el objetivo de establecer bases de colaboración técnica, académica y científica entre el Instituto Tecnológico de Durango (ITD) del Tecnológico Nacional de México (TecNM) y la Facultad de Medicina y Nutrición de la Universidad Juárez del Estado de Durango (UJED), este martes 19 de octubre se llevó a cabo importante firma del convenio, con el cual ambas instituciones educativas fortalecerán relaciones en beneficio de la comunidad estudiantil universitaria y el área de investigación de la Unidad de Posgrado, Investigación y Desarrollo Tecnológico (UPIDET) del ITD.

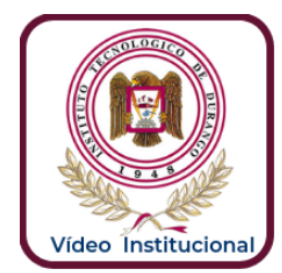

\section{Leer más}

Figura 1. Sistemas de gestión en el Instituto Tecnológico de Durango.

Nota: Sistemas de gestión certificados en el Instituto Tecnológico de Durango, sistemas de gestión. Adaptada de ITdurango, 2021 (https://www.itdurango.edu.mx).

Además, en la actualidad se cuenta con un sistema de gestión ambiental en formato multisitios. Es por ello, que a partir de ese sistema se sustenta la propuesta de integración de los sistemas, resultado en que, además del sistema de gestión de calidad se tiene el sistema de gestión ambiental. Según Uribe (2008) indica que el "despliegue del SGA (sistema de gestión ambiental) es un factor clave, porque permite que el recurso humano de la organización se concientice de sus responsabilidades, de los objetivos del sistema y de cómo comprometerse para su éxito" (p. 92).

Actualmente el Tecnológico tiene declarado el cumplimiento de la normatividad por parte del TecNM, y con ello se consigue establecer en todas las instituciones que dependen de este organismo. Y como cada uno de los sistemas de gestión, tienen declarado mapas de procesos que permiten entender las entradas y salidas de los procedimientos que se manejan. En la figura 2 se observa el mapa de procesos que se tiene para el SGA, el cual, si bien, declara los procedimientos y su interrelación, no lo hace desde un punto de vista integral. 


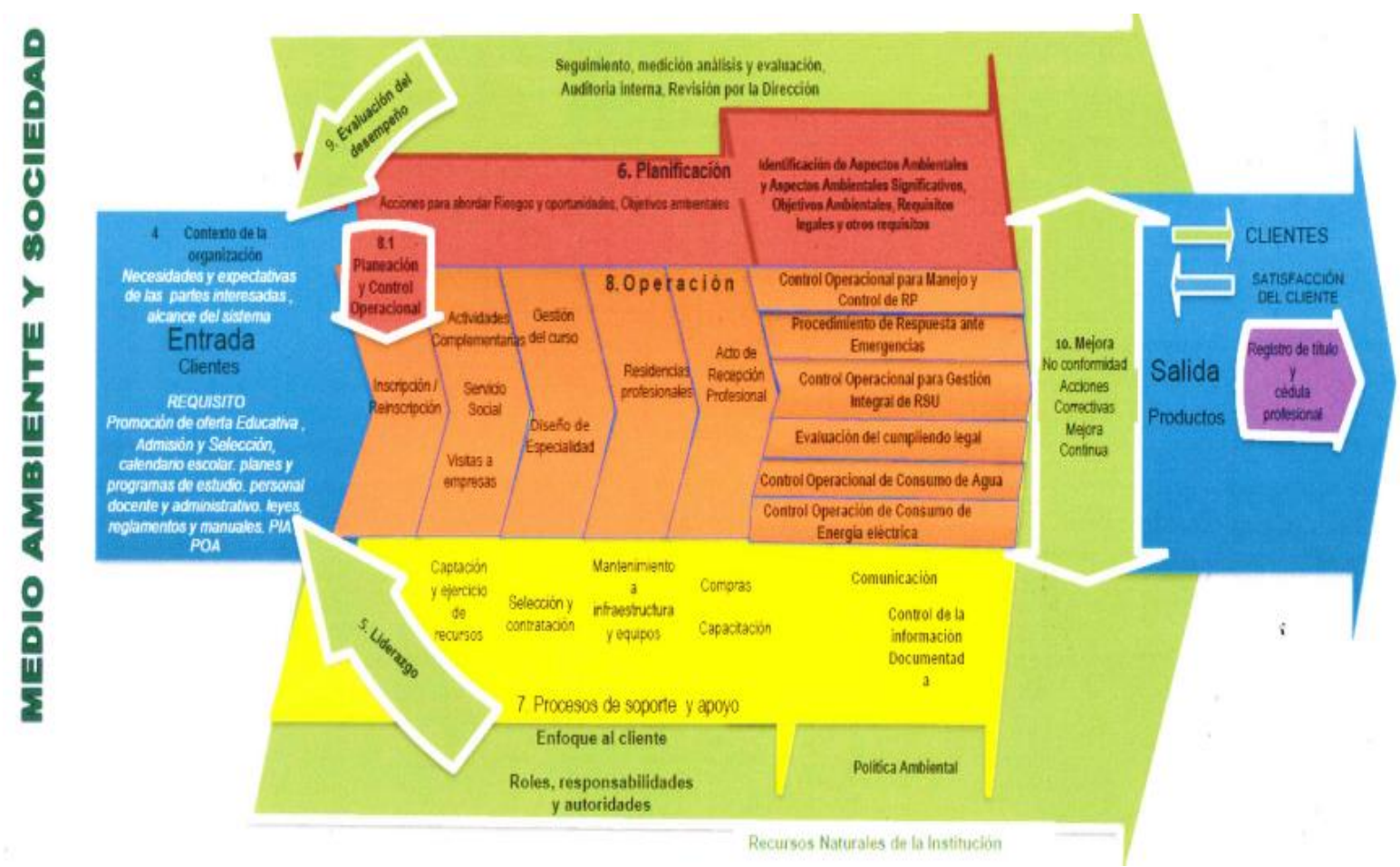

Figura 2. Mapa de procesos del sistema de gestión ambiental

Nota: Mapa de procesos del sistema de gestión ambiental del Tecnológico Nacional de México. Adaptada de TecNM, 2021 (https://www.tecnm.mx).

Además, en este sistema de gestión considera los aspectos ambientales significativos básicos tales como:

- Agua

- Energía

- Residuos sólidos urbanos

- Residuos peligrosos

Es por ello que quizá se cuestione, ¿porque es necesario migrar a un sistema integrado si actualmente ya se tienen dos sistemas de gestión bien establecidos y certificados? Se busca el sistema de gestión integrado porque: "un sistema estructurado de gestión, integrado con la actividad de gestión total de la organización, que incluye la estructura organizativa, la planificación de las actividades, las responsabilidades, las prácticas, los procedimientos, los procesos y los recursos para desarrollar, implantar, llevar a efecto, revisar y mantener al día los compromisos en materia de protección medioambiental que suscribe la organización" (Rey, 2008, p. 2). Así que, en la búsqueda de hacer una gestión total, se busca incorporar una mayor cantidad de normas necesarias para el quehacer educativo integral. El cuál permita involucrar a tres normas ISO más, además de las normas con las que ya se cuenta. En la figura 3 se agrupan las cinco normas que se buscan integrar. 

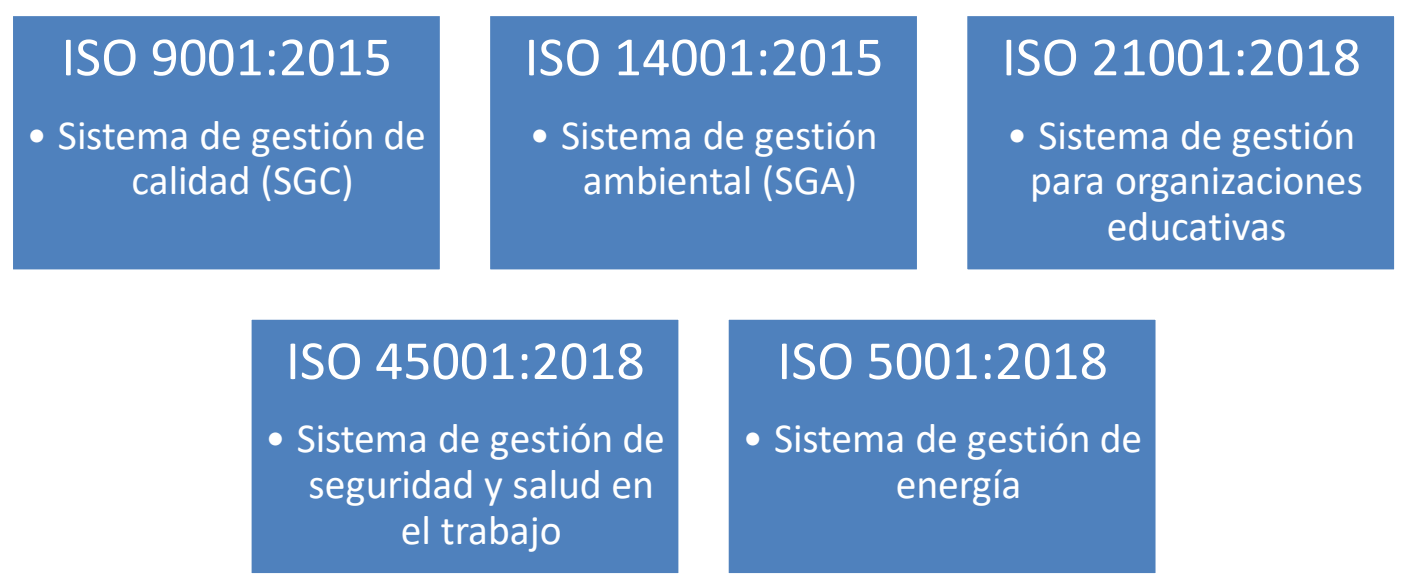

Figura 3. Normas elegidas para el sistema de gestión integrado.

Fuente: García, O. (2021)

¿Qué razones llevan a pensar en incorporar las normas de sistemas de gestión para organizaciones educativas, de gestión energética, así como la norma de gestión seguridad y salud en el trabajo? Es una realidad, que las escuelas se vieron afectadas como todas las empresas por la pandemia ocasionada en el pasado año de 2020, por el COVID-19, es por ello más que necesario "una correcta gestión en las organizaciones educativas siempre será fundamental, pero más aún en circunstancias excepcionales". Por lo que se debe aprovechar el momento de coyuntura generado por la pandemia para asegurar la gestión en el sector educativo a nivel licenciatura.

Por otra parte, con referencia a la necesidad de la implantación de la norma ISO 45001:2018, según Terán $(2012$, p. 8), es necesario contar con "un sistema de gestión de seguridad y salud ocupacional, que permita el control de la seguridad de sus procesos y la protección de la salud de sus trabajadores; logrando un mayor respaldo para la empresa y contribuyendo a un mejor desempeño y mayores beneficios".

Y, por último, en el caso de la norma de gestión energética, en México la ISO 50001 será una herramienta que permitirá la administración eficiente de la energía de forma permanente, que, de manera planeada, permita medir o estimar el consumo para realizar una mejora continua, en lugar de acciones de eficiencia energética aisladas (Acoltzi, 2011, p. 114). Al ser una de la normatividad más exigente y a la vez demanda un historial bastante detallado de la energía. Según Acoltzi (2011, p. 114) "Se debe conocer cuánta y dónde se está usando la energía, cuáles son los usos significativos, los aspectos que incluyen y/o la necesidad de realizar diagnósticos energéticos enfocados en la optimización del sistema". Es por ello que por medio de la técnica de revisión de documentos, principalmente requisiciones, es que se determinan los tipos de energía utilizados por cada departamento del Instituto y posteriormente se conjuntaron en los tipos de energía utilizados en todo el Instituto, encontrándose los siguientes mostrados en la figura 4. 


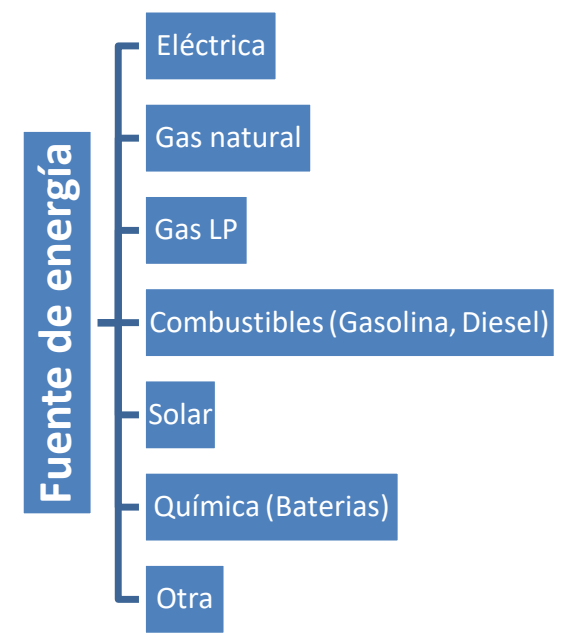

Figura 4. Fuentes de energía identificadas en el Instituto Tecnológico de Durango. Fuente: García, O. (2021)

Esta norma en lo particular exige que la revisión energética incluya el análisis y el uso de las fuentes de energía ya determinadas, para que pueda estimar, medir o calcular el uso y consumo presente, pasado y futuro de la energía. Dicho análisis deberá estandarizarse para estar en posibilidad de identificar el uso más relevante y con ello, generar un plan estratégico para reducir el impacto del uso de la energía en el aspecto más significativo de uso en la institución. (Nordelo, 2013, p. 12). Así que, en el análisis en plenaria inicialmente se identificaron 18 usos de la energía, los cuales posteriormente fueron unificados y definidos en los siguientes 8 usos de la energía, mostrados en la figura 5.

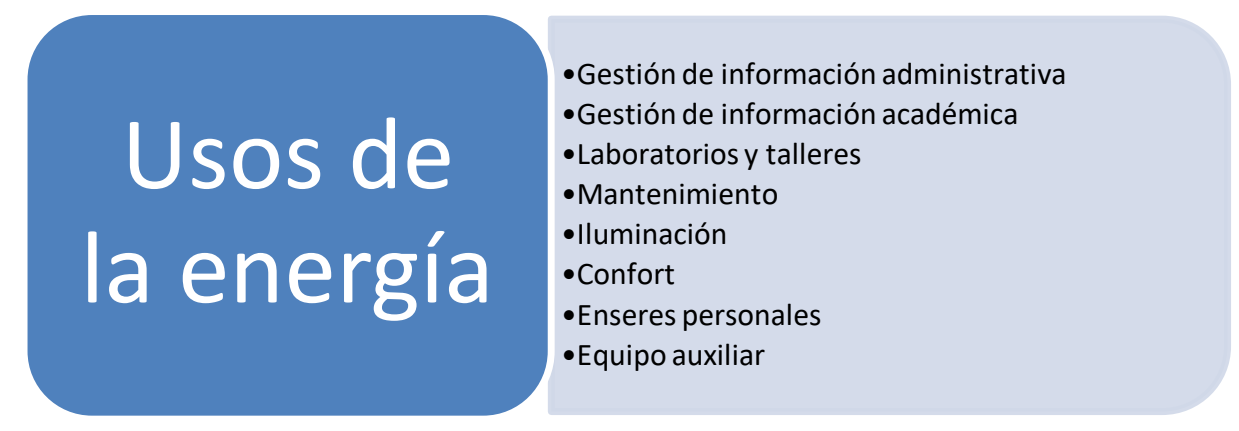

Figura 5. Usos de la energía utilizada en el Instituto Tecnológico de Durango. Fuente: García, O. (2021)

Una vez identificadas, se procederá al levantamiento de la información de los diferentes instrumentos consumidores de energía. De seguir así, en unos cuantos meses se tendrá la información suficiente y necesaria para iniciar con el proyecto de integración de los sistemas de gestión.

Sin embargo, ¿Qué beneficios otorga la integración de sistemas para una organización educativa? Un sistema de gestión es una estructura probada para la gestión y mejora continua de las políticas, los procedimientos y procesos de la organización. En la actualidad las empresas se enfrentan a muchos retos, y son precisamente los sistemas de gestión, los que van a permitir aprovechar y desarrollar el potencial existente en la organización. Además, tal 
como señala Heras (2007, p. 156), “el éxito en la difusión de tantos estándares de gestión, ha llevado a las organizaciones a que su implementación de pro fruto un único sistema de gestión integrado". Teniendo esto en cuenta es importante continuar con la implementación del sistema.

Después de haber realizado el primer paso, como segunda etapa, la parte directiva del instituto tiene pleno convencimiento de los beneficios de la integración del sistema, esto pensando en los siguientes beneficios:

- Menor cantidad de auditorias

- Uso efectivo del recurso económico y humano

- Un único sistema más ágil y ligero

Para el tercer paso se ha elegido a una persona como la encargada fundamental del proceso, quien ha recibido toda la autoridad para seleccionar un equipo de 25 personas de las diferentes áreas de la institución, se han determinado los grupos de trabajo para cada una de las normas y se ha comenzado con un proceso de conocimiento sobre las normas elegidas.

Quedando pendiente la realización y término de los pasos cuatro y cinco de la metodología seguida.

\section{RESULTADOS}

Actualmente se ha terminado la fase de planeación teniendo como tiempo establecido de 6 a 8 meses para la implantación del sistema integrado.

Se identificó la necesidad de capacitar al grupo de sistema de gestión y cuerpo directivo en la normatividad elegida para comenzar con la concientización de la apropiación de los sistemas. Se realizó una revisión exhaustiva de los procedimientos del sistema de gestión de calidad y de ambiental. La revisión se realizó en 6 procesos.

En primer lugar, en el proceso académico con los siguientes procedimientos: inscripción, reinscripción, gestión del curso, residencia profesional y sustentación de acto de recepción profesional. Como resultado del análisis se han añadido a este proceso los siguientes procedimientos: tutorías, actividades complementarias, control de prácticas, especialidad, actualización y formación docente.

En el proceso administrativo se actualizaron el instructivo de compras, captación de ingresos, ambiente de trabajo, selección de personal, mantenimiento preventivo y correctivo. Proponiendo que se añada este proceso la capacitación y desarrollo administrativo.

Para el procedimiento de vinculación se actualizaron los tres procedimientos, visitas a empresas, servicio social, promoción cultural y deportiva. En el proceso de calidad se actualiza quejas y sugerencias, auditoria de servicios, salida no conforme, revisión por la dirección, control de la información documenta y gestión de riesgos. Añadiéndose al proceso, un procedimiento único para la evaluación docente.

Para el proceso de planeación se actualiza POA y PTA, y se añaden participación y consulta, así como centro de información. Se actualizan los procedimientos de identificación de aspectos ambientales, los controles operativos de agua, energía, residuos sólidos urbanos y residuos peligrosos. Se crea el proceso de gestión de la energía 
Y se tiene como tarea posterior la creación de los procesos de las normas de organización educativa, además, de los procedimientos de gestión de la seguridad y salud en el trabajo.

\section{CONCLUSIONES}

Se tienen bien definido el alcance del Sistema de Gestión Integrado, lo que permite que todas las acciones y trabajos estén bien dirigidas.

El personal de Instituto está comprometido con el Sistema de Gestión Integrado, en las capacitaciones se ha tenido la participación activa tanto de los veintisiete miembros del Comité de Innovación y Calidad, como de los veinticuatro miembros del Comité del Sistema de Gestión Integrado, el primero conformado por los directivos de la institución y el segundo por trabajadores docentes y administrativos.

Se han revisado los procedimientos de los procesos del sistema de gestión de calidad, encontrando que se tienen significativas mejoras en la automatización de dichos procedimientos, impactando de manera positiva en la percepción de las partes interesadas.

\section{BIBLIOGRAFÍA}

Acoltzi, H., \& Pérez, H. (2011). ISO 50001, Gestión de energía. Boletín IIE, 114.

Castrillón Mendoza, R. D. P., González Hinestroza, A. J., Quispe Oqueña, E. C., \& Fandiño Gutiérrez, D. (2014). Metodología para la implementación del sistema de gestión integral de la energía: fundamentos y casos prácticos. Programa Editorial Universidad Autónoma de Occidente.

Gallego Navarro, T. (2013). Gestión integral. Unión de Editoriales Universitarias Españolas.

Gómez, J. A. (2020). ISO 21001, herramienta de gestión en el sector educativo. AENOR: Revista de la normalización y la certificación, (359), 21-30.

Heras Saizarbitoria, I., Bernardo Vilamitjana, M., \& Casadesus Fa, M. (2007). La integración de sistemas de gestión basados en estándares internacionales: resultados de un estudio empírico realizado en la CAPV. (C) Revista de Dirección y Administración de Empresas = Enpresen Zuzendaritza eta Administrazio Aldizkaria, 2007, núm. 14, p. 155-174.

Hurtado, F. A. A., Vélez, R. E. B., \& de los Ríos, J. A. V. (2008). Sistema de gestión integral. Una sola gestión, un solo equipo. Universidad de Antioquia.

Nordelo, A. B., \& Caminos, C. (2013). Recomendaciones Metodológicas para la Implementación de Sistemas de Gestión de la Energía según la Norma ISO 50001. Editorial Universo Sur ISBN, 978-959.

Rey, C. (2008). Sistema de Gestión Ambiental. Master en Ingeniería y gestion Medio ambiental, 7. EOI.

Reyes Tapia, C. X., \& Tapia Encalada, J. G. (2014). Diseño del Sistema de Gestión de Energía en la Escuela Politécnica Nacional, basado en la norma ISO 50001, caso de estudio: edificación de la Facultad de Ingeniería Civil y Ambiental (Bachelor's thesis, Quito, 2014.).

Serrano, A. T. I. (2018). Control interno y sistema de gestión de calidad: Guía para su implantación en empresas públicas y privadas. Ediciones de la U. 
Terán Pareja, I. S. (2012). Propuesta de implementación de un sistema de gestión de seguridad y salud ocupacional bajo la norma OHSAS 18001 en una empresa de capacitación técnica para la industria.

Triveño Ramos, P. L. (2020). Diseño de un Sistema de Gestión en Seguridad y Salud en el trabajo basado en la Norma ISO 45001 de la "Escuela Profesional de Ingeniería Mecánica de la Universidad Nacional de San Antonio Abad del Cusco", 2018.

Uribe, R. P., \& Bejarano, A. (2008). Sistema de gestión ambiental: Serie ISO 14000. revista Escuela de Administración de Negocios, (62), 89-105.

Valencia-Duque, F. J., \& Orozco-Alzate, M. (2017). Metodología para la implementación de un Sistema de Gestión de Seguridad de la Información basado en la familia de normas ISO/IEC 27000. Revista Ibérica de Sistemas e Tecnologías de Informação, (22), 73.

Vílchez, E. J. G. (2008). Ventajas de la implantación de un sistema de gestión ambiental. Técnica industrial, 273, 41. 